\title{
Study of the Dynamic Characteristics of Some Circuit Optimization Strategies
}

\author{
ALEXANDER ZEMLIAK \\ Department of Physics and Mathematics \\ Puebla Autonomous University \\ MEXICO \\ Institute of Technical Physics \\ National Technical University of Ukraine \\ UKRAINE
}

\begin{abstract}
The work explores the possibility of a significant reduction in processor time required to optimize analog circuits. For this, we can reformulate and generalize the problem of chain optimization based on the approaches of optimal control theory. The analog circuit design process is formulated as a dynamic controlled system. A special control vector has been defined to redistribute the computational cost between circuit analysis and parametric optimization. This redistribution significantly reduces CPU time. The task of designing a circuit for the minimum processor time can be formulated as a classical optimal control problem while minimizing some functional. The introduction of the concept of the Lyapunov function of a controlled dynamic system made it possible to use it to analyse the main characteristics of the design process. An analysis of the behaviour of a special function, which is a combination of the Lyapunov function and its time derivative, made it possible to compare various design strategies and select the best ones that are executed in minimal CPU time.
\end{abstract}

Key Words: - Minimal-time system design, control theory application, Lyapunov function.

Received: November 8, 2019. Revised: March 4, 2020. Accepted: April 8, 2020. Published: April 30, 2020.

\section{Introduction}

Reducing computer time in the design of large systems is one of the sources of overall improvement in design quality. In addition to the traditionally used ideas and methods of a sparse matrix and decomposition methods [1-5], other methods for reducing the total CPU time in the design of systems were previously proposed [6-8]. A generalized approach to the design of analog systems based on the formulation of control theory was developed in some previous works, for example [9-10]. This approach serves to determine the system design algorithm for the minimum CPU time. On the other hand, this approach makes it possible to analyse the system design process with great clarity when moving along a trajectory in the design space. The main concept of this approach is the introduction of special management functions, which, on the one hand, generalize the design process, and on the other hand, make it possible to control the design process to achieve the optimal point of the cost function for minimal computer time. This possibility appears because an almost infinite number of different design strategies exist within the framework of the proposed approach. Different design strategies have different numbers of operations and different CPU times. Within this concept, a traditional design strategy is just one representative of a vast array of different design strategies. As shown in [9], the potential gain in computer time, which can be obtained using a new formulation of the design problem, increases with increasing size and complexity of the system. However, this gain can be realized in practice only when the optimal algorithm has already been created and it implements the optimal design strategy.

This paper compares various design strategies in terms of CPU time and stability. This analysis is one of the main steps to constructing an optimal algorithm. The main tool on this path is the introduction of the Lyapunov function of the circuit optimization process.

\section{Problem formulation}

The design process for any analog system design can be defined in discrete form [9] as the problem of the generalized cost function $F(X, U)$ minimization by means of the system (1) with the constraints (2):

$$
\begin{gathered}
x_{i}^{s+1}=x_{i}^{s}+t_{s} \cdot f_{i}(X, U), \quad i=1,2, \ldots, N \\
\left(1-u_{j}\right) g_{j}(X)=0, \quad j=1,2, \ldots, M
\end{gathered}
$$


where $X \in R^{N}, X=\left(X^{\prime}, X^{\prime \prime}\right), X^{\prime} \in R^{K}$ is the vector of the independent variables and the vector $X^{\prime \prime} \in R^{M}$ is the vector of dependent variables $(N=K+M)$, $g_{j}(X)$ for all $j$ presents the network model, $s$ is the iterations number, $t_{s}$ is the iteration parameter, $t_{s} \in R^{1}, \quad H \equiv H(X, U)$ is the direction of the generalized cost function $F(X, U)$ decreasing, $U$ is the vector of the special control functions $U=\left(u_{1}, u_{2}, \ldots, u_{m}\right)$, where $u_{j} \in \Omega ; \quad \Omega=\{0 ; 1\}$. The functions $f_{i}(X, U)$ for example for the gradient method are defined as:

$$
\begin{gathered}
f_{i}(X, U)=-\frac{\delta}{\delta x_{i}} F(X, U), i=1,2, \ldots, K \\
f_{i}(X, U)=-u_{i-K} \frac{\delta}{\delta x_{i}} F(X, U)+\frac{\left(1-u_{i-K}\right)}{t_{s}}\left\{-x_{i}^{s}+\eta_{i}(X)\right\}, \\
i=K+1, K+2, \ldots, N
\end{gathered}
$$

where the operator $\frac{\delta}{\delta x_{i}}$ hear and below means $\frac{\delta}{\delta x_{i}} \varphi(X)=\frac{\partial \varphi(X)}{\partial x_{i}}+\sum_{p=K+1}^{K+M} \frac{\partial \varphi(X)}{\partial x_{p}} \frac{\partial x_{p}}{\partial x_{i}}, x_{i}^{s}$ is equal to $x_{i}(t-d t) ; \quad \eta_{i}(X)$ is the implicit function $\left(x_{i}=\eta_{i}(X)\right)$ that is determined by the system (2). The generalized cost function $F(X, U)$ can be defined as:

$$
F(X, U)=C(X)+\psi(X, U)
$$

where $C(X)$ is the non negative cost function of the design process, and $\psi(X, U)$ is the additional penalty function:

$$
\psi(X, U)=\frac{1}{\varepsilon} \sum_{j=1}^{M} u_{j} \cdot g_{j}^{2}(X)
$$

This formulation of the design process permits the redistribution of the computer time expense between the solution of problem (2) and the optimization procedure (1) for the function $F(X, U)$. The control vector $U$ is the main tool for the redistribution process in this case. Practically an infinite number of the different design strategies are produced because the vector $U$ depends on the optimization procedure current step. The problem of the optimal design strategy is formulated now as the typical problem for the functional minimization of the control theory. The functional that needs to minimize is the total CPU time $T$ of the design process. This functional depends directly on the operations number and on the design strategy that has been realized. The main difficulty of this definition is unknown optimal dependencies of all control functions $u_{j}$. It is necessary to find the optimal behavior of the control functions $u_{j}$ during the design process to minimize the total design computer time.

The idea of the system design problem formulation as the functional minimization problem of the control theory is not depend of the optimization method and can be embedded into any optimization procedures. In this paper the gradient method is used, nevertheless any optimization method can be used as shown in [9].

Now the process for analog circuit design is formulated as a dynamic controllable system. The minimal-time design process can be defined as the dynamic system with the minimal transition time. So, we need to find the special conditions to minimize the transition time for this dynamic system.

\section{Definition of the Lyapunov function}

On the basis of the analysis in previous section we can conclude that the minimal-time algorithm has one or some switch points in control vector where the switching is realize among different design strategies. As shown in [10] it is necessary to switch the control vector from like modified traditional design strategy (MTDS) to like traditional design strategy (TDS) with some adjusting. Some principal features of the time-optimal algorithm were determined previously. These are: 1) an additional acceleration effect that appeared under special circumstances [10]; 2) the start point special selection outside the separate hyper-surface to guarantee the acceleration effect, at least one negative component of the start value of the vector $X$ is can be recommended for this; 3) analysis of stability and the structure of more perspective strategies. The two first problems were discussed in [10-11]. The third problem is discussed in the present paper.

The main problem of the time-optimal algorithm construction is unknown optimal sequence of the switch points during the design process. We need to define a special criterion that permits to realize the optimal or quasi-optimal algorithm by means of the optimal switch points searching. A Lyapunov function of dynamic system serves as a very informative object to any system analysis in limits of the control theory. We propose to use a Lyapunov function of the design process to detect the optimal 
algorithm, particularly for the optimal switch points searching. The Lyapunov function properties can help us to solve this problem.

There is a freedom of the Lyapunov function choice because of a non-unique form of this function. Let us define the Lyapunov function of the design process (1)-(5) by the following expression:

$$
V(X)=\sum_{i}\left(x_{i}-a_{i}\right)^{2}
$$

where $a_{i}$ is the stationary value of the coordinate $x_{i}$, in other words the set of all the coefficients $a_{i}$ is the main objective of the design process. The function (6) satisfies all of the conditions of the standard Lyapunov function definition for the variables $y_{i}=x_{i}-a_{i}$. In fact the function

$$
V(Y)=\sum_{i} y_{i}^{2}
$$

is the piecewise continue. Besides there are three characteristics of this function: i) $V(Y)>0$, ii) $V(0)=0$, and iii) $V(Y) \rightarrow \infty$ when $\|Y\| \rightarrow \infty$.

Inconvenience of the formulas (6) or (7) is an unknown point $A=\left(a_{1}, a_{2}, \ldots, a_{N}\right)$, because this point can be reached at the end of the design process only. We can use this form of the Lyapunov function if we already found the design solution someway. On the other hand, it is very important to control the stability of the design process during the optimization procedure. In this case we need to construct other form of the Lyapunov function that doesn't depend on the unknown stationary point. Let us define two new forms of the Lyapunov function by the next formulas:

$$
\begin{gathered}
V(X, U)=[F(X, U)]^{r} \\
V(X, U)=\sum_{i}\left(\frac{\partial F(X, U)}{\partial x_{i}}\right)^{2}
\end{gathered}
$$

where $F(X, U)$ is the generalized cost function of the design process. The formula (8) can be used when the general cost function is non-negative and has zero value at the stationary point $A$. Other formula can be used always because all derivatives $\partial F / \partial x_{i}$ are equal to zero in the stationary point $A$. So, the function $V$ for both formulas has properties: $V(A, U)=0, V(X, U)>0$ for all $X$ and at last, this function increases in a sufficient large neighborhood of the stationary point. Besides, the function $V$ is the function of the vector $U$ too, because all coordinates $x_{i}$ are the functions of the control vector $U$.

We can define now the design process as a transition process for controllable dynamic system that can provide the stationary point (optimal point of the design procedure) during some time. The problem of the time-optimal design algorithm construction can be formulated now as the problem of the transition process searching with the minimal transition time. There is a well-known idea [12-13] to minimize the time of the transition process by means of the special choice of the right hand part of the principal system of equations; in our case these are the functions $f_{i}(X, U)$. It is necessary to change the functions $f_{i}(X, U)$ by means of the control vector $U$ selection to obtain the maximum speed of the Lyapunov function decreasing (the maximum absolute value of the Lyapunov function time derivative $\dot{V}=d V / d t)$.

\section{Stability analysis}

Normally the time derivative of the Lyapunov function is non-positive for the stable processes. The design process is stable if the Lyapunov function time derivative is negative. On the other hand, the direct method of Lyapunov gives the sufficient stability conditions but not necessary [12-13], so the process loses the stability (or not loses) if this derivative becomes positive. The stability of the different design strategies for two-stage transistor amplifier of Fig. 1 was analyzed by the Lyapunov direct method. This network is characterized by five dependent parameters and the control vector includes five control functions: $U=\left(u_{1}, u_{2}, u_{3}, u_{4}, u_{5}\right)$. The structural basis consists of 32 design strategies. The Ebers-Moll static model of the transistor has been used [14].

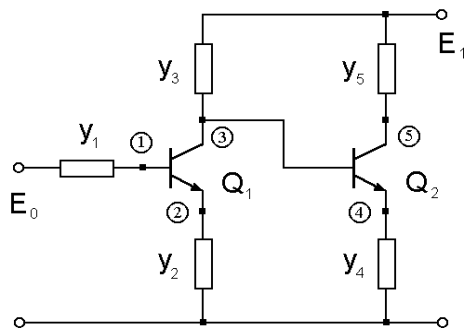

Fig. 1 Two-stage transistor amplifier

The Lyapunov function time derivative $d V / d t$ is a negative for all trajectories on the initial part of the design $\mathrm{n}$ process; i.e. all admissible strategies are stable at the beginning. It is supposed that the integration step is sufficiently small. However, when 
the current point of the trajectory gets to the $\mathcal{E}$ neighborhood of the stationary point $A$, the strategies can lose the stability because the time derivative of the Lyapunov function becomes positive. It means that all trajectories of this group do not guarantee the convergence from the $\varepsilon$-neighborhood. In fact, each of the trajectories of this group has own critical $\varepsilon$ neighborhood, which defines the maximum achievable precision. Another consideration is important too: the design process convergence slow down strongly before the $\varepsilon$-neighborhood reaching for all strategies of this group. It means that the derivative $d V / d t$ is the negative but very small on the absolute value. The critical values of the $\varepsilon$ neighborhood of some design trajectories for the twostage transistor amplifier are shown in Table 1.

Table 1. Critical $\varepsilon$ value for two-stage amplifier

\begin{tabular}{|r|c|r|r|r|}
\hline $\mathrm{N}$ & $\begin{array}{l}\text { Control vector } \\
\mathrm{U}(\mathrm{u} 1, \mathrm{u} 2, \mathrm{u} 3, \mathrm{u} 4, \mathrm{u} 5)\end{array}$ & $\begin{array}{l}\text { Iterations } \\
\text { number }\end{array}$ & $\begin{array}{l}\text { Computer } \\
\text { time }(\mathrm{sec})\end{array}$ & $\begin{array}{l}\text { Critical value of } \\
\mathcal{E} \text {-neighborhood }\end{array}$ \\
\hline 1 & $(00000)$ & 3177 & 7,25 & $2,78 \mathrm{E}-08$ \\
\hline 2 & $(00001)$ & 3074 & 8,02 & $3,36 \mathrm{E}-07$ \\
\hline 3 & $(00011)$ & 11438 & 26,36 & $8,18 \mathrm{E}-07$ \\
\hline 4 & $(00101)$ & 799 & 1,16 & $9,38 \mathrm{E}-09$ \\
\hline 5 & $(00110)$ & 1798 & 2,6 & $1,61 \mathrm{E}-08$ \\
\hline 6 & $(01011)$ & 43431 & 76,89 & $3,16 \mathrm{E}-05$ \\
\hline 7 & $(01100)$ & 1378 & 2,25 & $1,67 \mathrm{E}-08$ \\
\hline 8 & $(01101)$ & 571 & 0,72 & $6,83 \mathrm{E}-09$ \\
\hline 9 & $(01110)$ & 1542 & 2,03 & $2,05 \mathrm{E}-08$ \\
\hline 10 & $(10011)$ & 11839 & 21,37 & $1,68 \mathrm{E}-05$ \\
\hline 11 & $(10100)$ & 2097 & 3,57 & $5,47 \mathrm{E}-07$ \\
\hline 12 & $(10110)$ & 6026 & 8,31 & $4,94 \mathrm{E}-07$ \\
\hline 13 & $(11100)$ & 6602 & 8,84 & $7,41 \mathrm{E}-07$ \\
\hline 14 & $(11101)$ & 935 & 0,71 & $1,33 \mathrm{E}-08$ \\
\hline 15 & $(11110)$ & 2340 & 2,31 & $1,62 \mathrm{E}-07$ \\
\hline 16 & $(11111)$ & 1502 & 0,38 & $1,09 \mathrm{E}-08$ \\
\hline
\end{tabular}

The termination of the design process has been defined by means of the special criterion: the time derivative $\dot{V}$ has positive value in the set of positive measure. The optimization process was provided on the basis of the formula (1) with variable step. As a result the significant variations of the derivative value $\dot{V}$ have been observed. We average the derivative values for 30 integration steps to smooth these variations.

The analysis of results in Table 1 gives the next regularities: there is a correlation between processor design time and a critical $\varepsilon$-neighborhood. The processor design time is lesser for the strategies that have lesser critical $\mathcal{E}$-neighborhood. We can rank all the strategies of Table 1 in the order of computer time or in the order of critical $\varepsilon$-neighborhood. The results of this ranking are presented in Table 2.
Table 2. Strategy ranking for two-stage amplifier

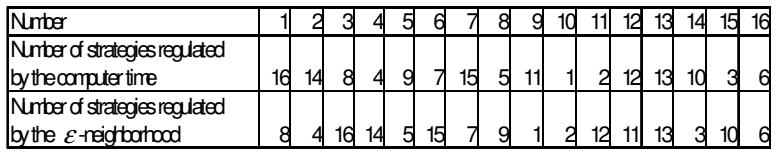

The places of each strategy, which defined by two different methods of regulation, are differed very small. This place is the same for two strategies (13 and 6). The difference in one place appears seven times, the difference in two places appears four times, and the difference in three places appears three times. The average value of this difference is 1.5 . We can consider that the correlation between a computer time and a critical $\varepsilon$-neighborhood is acceptable. From the other hand the critical $\varepsilon$-neighborhood values were obtained by the analysis of the Lyapunov function and its derivative. So, we can state that there is a close relation between the computer time and the properties of the Lyapunov function.

The analysis of the three-stage amplifier of Fig. 2 shows very similar results. The critical value of $\varepsilon$ neighborhood of some design trajectories for the three-stage transistor amplifier is shown in Table 3.

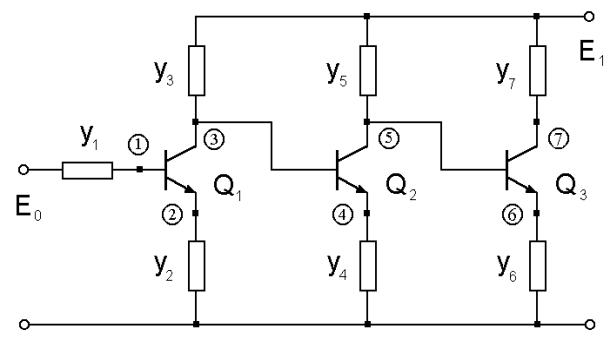

Fig. 2 Three-stage transistor amplifier

Table 3. Critical $\varepsilon$ value for three-stage amplifier

\begin{tabular}{|c|c|c|c|c|}
\hline & 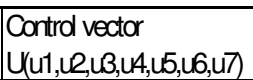 & $\begin{array}{l}\text { Iterations } \\
\text { number }\end{array}$ & $\begin{array}{l}\text { Computer } \\
\text { time (sec) }\end{array}$ & $\begin{array}{l}\text { Oritical value of } \\
\mathcal{E} \text {-neighborhood }\end{array}$ \\
\hline 1 & $(0000000)$ & 9106 & 45,75 & $7,27 \mathrm{E}-07$ \\
\hline 2 & $(0010110)$ & 1844 & 8,63 & $1,91 \mathrm{E}-07$ \\
\hline 3 & $(0011100)$ & 3067 & 15,65 & $6,7 \pi \mathrm{E}-07$ \\
\hline 4 & $(0011111)$ & 647 & 1,87 & $4,73 E-09$ \\
\hline 5 & $(0110101)$ & 627 & 1,42 & $4,69 E-09$ \\
\hline 6 & $(0110110)$ & 1513 & 4,01 & $5,62 \mathrm{E}-07$ \\
\hline 7 & $(0110111)$ & 643 & 1,21 & $9,07 E-09$ \\
\hline 8 & $(0111110)$ & 3229 & 7,30 & $7,54 \mathrm{E}-07$ \\
\hline 9 & $(1010101)$ & 2069 & 4,06 & $1,43 E-07$ \\
\hline 10 & $(1011101)$ & 1657 & 2,53 & $2,13 E-07$ \\
\hline 11 & $(1110101)$ & 1477 & 2,09 & $1,33 E-07$ \\
\hline 12 & $(1110110)$ & 3931 & 6,48 & 9,21E-07 \\
\hline 13 & $(1111100)$ & 3626 & 7,85 & $6,25 \mathrm{E}-07$ \\
\hline 14 & $(1111101)$ & 1793 & 2,03 & $2,12 \mathrm{E}-07$ \\
\hline 15 & $(1111110)$ & 2345 & 3,51 & $208 E-07$ \\
\hline 16 & $(1111111)$ & 2149 & 0,61 & $7,59 E-08$ \\
\hline
\end{tabular}


As for the first example we can formulate the rule - the less value of the critical $\varepsilon$ - neighborhood for some strategy provoke the less value of the computer time for this strategy. The regulations of all the strategies of Table 3 in order of the computer time and in order of the critical $\varepsilon$-neighborhood are presented in Table 4.

Table 4. Strategy ranking for three-stage amplifier

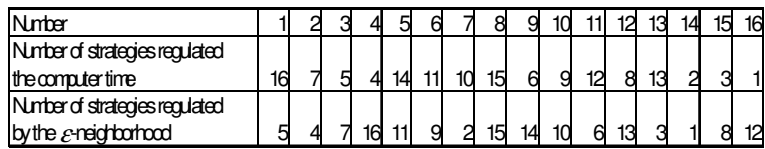

The places of each strategy, which defined by two different methods of regulation, are differed small. The average value of this difference is 2.6 . We can conclude that the Lyapunov function can serves as the informative source for the searching of the perspective strategies that have the minimal computer time.

\section{Analysis of structural basis}

The analysis of trajectory stability and the correlation between a Lyapunov function and CPU time can be done better by means of a special function. We can define more informative function as a time derivative of the Lyapunov function relatively the Lyapunov function: $W=\dot{V} / V$. In this case we can compare the different design strategies by means of behavior of the function $W(t)$.

The results of the analysis of some optimization strategies from the structural basis for the iterations number and computer time for two-stage amplifier are shown in Table 5.

The behavior of functions $V(t)$ and $W(t)$ were the main objects of the analysis and have been analyzed for some strategies that compose the structural basis of the general methodology. The behaviour of these functions that correspond to the data of the Table 5 is shown in Fig. 3. These graphs correspond to a time interval when the majority of the design strategies are

Table 5. Data of some strategies for circuit in Fig. 1

\begin{tabular}{|c|c|c|c|}
\hline $\mathrm{N}$ & $\begin{array}{l}\text { Control } \\
\text { vector }\end{array}$ & $\begin{array}{l}\text { Iterations } \\
\text { number }\end{array}$ & $\begin{array}{l}\text { Total design } \\
\text { time (sec) }\end{array}$ \\
\hline 1 & $\left(\begin{array}{lllll}0 & 0 & 0 & 0 & 0\end{array}\right)$ & 165962 & 299,56 \\
\hline 2 & $(000001)$ & 337487 & 737,55 \\
\hline 3 & $(00100)$ & 44118 & 68,87 \\
\hline 4 & $(001001)$ & 14941 & 19,06 \\
\hline 5 & $(00111)$ & 21971 & 22,03 \\
\hline 6 & 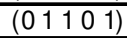 & 4544 & 4,56 \\
\hline 7 & $(10101)$ & 2485 & 1,65 \\
\hline 8 & $(10111)$ & 7106 & 3,57 \\
\hline 9 & $(11101)$ & 2668 & 1,32 \\
\hline 10 & $(11111)$ & 79330 & 10,11 \\
\hline
\end{tabular}




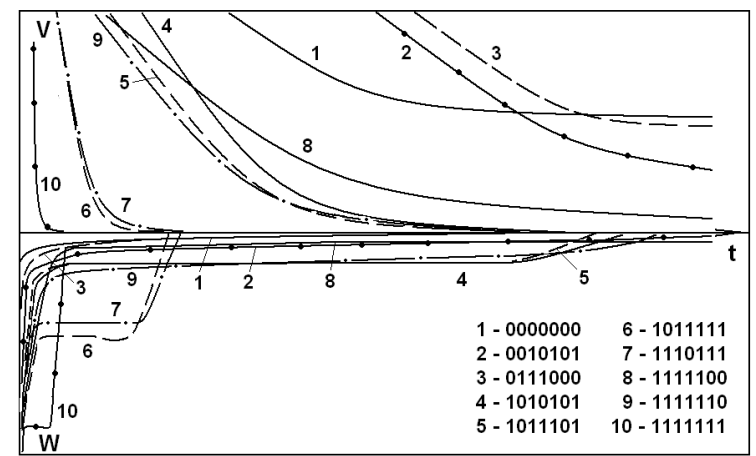

Fig. 4 Behaviour of the functions $V(t)$ and $W(t)$ for different design strategies during the design process for three-stage amplifier

These graphs correspond to a time interval when the majority of the design strategies are finished. The strategies 6,7 , and 10 have a minimal relative computer time because the function $W(t)$ for these strategies has a relatively large negative value during a long time of the design process in spite of the large value of Lyapunov function $V(t)$ in the initial time interval. On the contrary, the function $W(t)$ has a relatively small value for the strategies 1,2 and 3 . That is why these strategies have a large computer design time. Once again, we got a strong correlation between the behavior of the function $W(t)$ and processor time.

So, the main result of the analyzed examples can be formulated as follows: the behavior of the Lyapunov function $\mathrm{V}$ and the relative time derivative $\mathrm{W}$ determine the CPU time with confidence. This means that by analyzing the behavior of these functions, you can predict the CPU time for any design strategy. We could analyse the behaviour of the functions $V(t)$ and $W(t)$ for the initial time interval for some strategies, and based on this analysis we can predict strategies that have minimal computer design time.

\section{Conclusions}

The problem of the minimal-time design algorithm construction can be solved adequately on the basis of the control theory. The design process in this case is formulated as the controllable dynamic system. The Lyapunov function of the design process and its time derivative include the sufficient information to select more perspective design strategies from the infinite set of the different design strategies that exist into the generalized design methodology. A special function $W(t)$ was proposed for analyzing the behaviour of various optimization strategies. This function serves as the main criterion for comparing strategies and allows you to choose the most promising of them, providing the minimum CPU time. The obtained result will allow us to choose the best strategies in the construction of the optimal algorithm.

References:

[1] J. R. Bunch and D. J. Rose, (Eds), Sparse Matrix Computations, Acad. Press, N.Y., 1976.

[2] O. Osterby and Z. Zlatev, Direct Methods for Sparse Matrices, Springer-Verlag, N.Y., 1983.

[3] F. F. Wu, Solution of large-scale networks by tearing, IEEE Trans. Circuits Syst., Vol. CAS23, No. 12, pp. 706-713, 1976.

[4] A. Sangiovanni-Vincentelli, L. K. Chen and L. O. Chua, An efficient cluster algorithm for tearing large-scale networks, IEEE Trans. Circuits Syst., Vol. CAS-24, No. 12, pp. 709717, 1977.

[5] N. Rabat, A. E. Ruehli, G. W. Mahoney and J. J. Coleman, A survey of macromodeling, Proc. of the IEEE Int. Symp. Circuits Systems, pp. 139143, April 1985.

[6] I.S. Kashirskiy and I.K. Trokhimenko, General Optimization for Electronic Circuits, Kiev: Tekhnika, 1979.

[7] V. Rizzoli, A. Costanzo and C. Cecchetti, Numerical optimization of broadband nonlinear microwave circuits, IEEE MTT-S Int. Symp., Vol. 1, pp. 335-338, 1990.

[8] E. S. Ochotta, R. A. Rutenbar and L. R. Carley, Synthesis of high-performance analog circuits in ASTRX/OBLX, IEEE Trans. on CAD, Vol.15, No. 3, pp. 273-294, 1996.

[9] A.M. Zemliak, Design of Analog Networks by Control Theory Methods, Part 1, Theory, Radioelectronics and Communications Systems, Vol. 47, No. 5, 2004, pp. 11-17.

[10] A. Zemliak, Analog circuit optimization on basis of control theory approach, COMPEL: The International Journal for Computation and Mathematics in Electrical and Electronic Engineering, Vol. 33, No. 6, 2014. pp. 21802204.

[11] A. Zemliak, $\mathrm{T}$ Markina, Behaviour of Lyapunov's function for different strategies of circuit optimization, International Journal of Electronics, Vol. 102, No. 4, 2015, pp. 619-634.

[12] E.A. Barbashin, Introduction to the Stability Theory, Nauka, Moscow, 1967.

[13] N. Rouche, P. Habets and M. Laloy, Stability Theory by Liapunov's Direct Method, SpringerVerlag, N.Y, 1977.

[14] G. Massobrio and P. Antognetti, Semiconductor Device Modeling with SPICE, N.Y.: Mc. GrawHill, Inc., 1993. 